\title{
Collision of protons with carbon atoms of a graphene surface in the presence of adsorbed potassium
}

\author{
A. Iglesias-García ๑, ${ }^{*}$ M. A. Romero ๑, Evelina A. García, and E. C. Goldberg ๑ \\ Instituto de Física del Litoral (CONICET-UNL), Güemes 3450, S3000GLN Santa Fe, Argentina
}

(Received 21 May 2020; revised 17 July 2020; accepted 4 August 2020; published 8 September 2020)

\begin{abstract}
In this work we study the frontal collision of protons with the carbon atoms of a graphene surface with a low coverage of adsorbed potassium. It is aimed at the analysis of the effect of the adsorbates in both charge exchange and electron emission processes, when the binary collision occurs between the proton and a carbon atom of the surface. The frontal collision with the $\mathrm{K}$ adsorbate, already analyzed and discussed in a previous work, is compared with the frontal collision with different carbon neighbors. In the present work we studied the signals, due to the localized structures in the density matrix of the composed graphene plus potassium surface, that can be distinguished when the collision occurs either with the adsorbate, a nearby carbon atom, or a carbon atom that does not feel the presence of the adsorbate. The interacting system is described by the Anderson Hamiltonian which takes into account the electronic repulsion on the projectile site; the charge fractions, the energy distribution of electrons in the solid, and the electron emission after the collision are calculated by using the nonequilibrium Green-Keldysh functions formalism solved by the equation of motion method. In the binary collision with a carbon atom, the extended features of the band structure of graphene smooth the dependence of the projectile charge fractions on the incoming energy and notably decrease the negative ions formation. The localized structures of the density of matrix caused by the presence of the adsorbate are perceptible for scattered carbon atoms close to $\mathrm{K}$. The intense emission of low energy electrons obtained in the case of the scattering by potassium is fundamentally associated with the very localized K- $4 s$ empty band. This characteristic, although less marked, remain in the scattering by nearby carbon atoms, due to both the interaction with $\mathrm{K}$ along the projectile trajectory and the perturbed local density of states on the carbon atoms due to the adsorbate presence. In addition, the extended nature of the electronic structure of graphene allows for the emission of more energetic electrons.
\end{abstract}

DOI: 10.1103/PhysRevB.102.115406

\section{INTRODUCTION}

The ion scattering from metal surfaces covered with alkali atoms has been and continues to be a subject of interest that has motivated many experimental and theoretical works [1-18]. The adsorbates on a metal surface introduce local effects as the change in the density matrix of the adsorbatesurface system, and also nonlocal effects as the change of the surface work function. In the case of alkaline atoms adsorbed at small coverage, the work function diminishes due to the creation of a dipole layer on the surface [2]. Therefore, it is expected that a low coverage of alkali atoms affects the charge exchange and electron emission in the ion-surface scattering processes, and that the effects will be strongly dependent on the surface atom involved in the binary collision. In particular, Yu et al. in Ref. [16] present a detailed study of the local effect induced by the $\mathrm{Na}$ atoms adsorbed on $\mathrm{Cu}(111)$ at very low coverage. The $\mathrm{Na}$ atom and its three $\mathrm{Cu}$ atom neighbors tend to form a small cluster, the effect of the $\mathrm{Na}$ atom on the surface being localized. This result is closely related to the results obtained by the same authors in Ref. [15], where charge transfer studies in $\mathrm{H}^{-}$collisions with $\mathrm{Na} / \mathrm{Cu}(111)$ show that the $\mathrm{Na}$ adsorbates induce strong local perturbations in the electronic structure and potentials in their surroundings.

\footnotetext{
*adalberto.iglesias@ifis.santafe-conicet.gov.ar
}

As a consequence, the charge transfer processes between projectiles and surface are appreciably modified in the vicinity of adsorbates.

In a previous work [18] we analyzed the frontal collision of protons with a potassium atom adsorbed on a graphene surface. Apart from its technological applications, the interest in graphene is motivated by the singular characteristics of its electronic band structure which gives place to peculiar hybridizations with adsorbed alkaline atoms [19-24].

In Ref. [18] the resonant charge exchange between the proton and the composed surface graphene plus adsorbed potassium was described by an Anderson model that takes into account the three correlated charge states of the hydrogen projectile: positive, neutral, and negative. The surface density matrix includes the electronic repulsion in the valence states of $\mathrm{K}$ and also the hybridization of the $\mathrm{K}-3 p$ states with the graphene band states, together with the local changes in the density matrix on the surrounding $\mathrm{C}$ atoms induced by the presence of the $\mathrm{K}$ adatom. In this previous work we found that in a frontal collision with the $\mathrm{K}$ adatom, the $\mathrm{C}$ atoms practically do not participate in the charge exchange process. We also concluded that the pronounced oscillatory behavior of the projectile charge fractions with the incoming energy is caused by the correlated states of hydrogen interacting with the localized band states of the potassium adsorbed on graphene.

The present work, which can be considered a natural continuation of Ref. [18], is devoted to the analysis of the 
frontal collision of protons with carbon atoms nearby the $\mathrm{K}$ adsorbate. The importance of studying the effects caused by the presence of the potassium on the charge exchange and the electron emission processes when the binary collision is with a carbon atom, resides basically in the ability to identify the signals of a low concentration of impurities in the ion scattering process. The energy spectra of emitted electrons by a kinetic mechanism, calculated from the convolution of the holes and excited electrons distributions, is very sensitive to the details of the surface density of states, particularly to the localized features of the surface band structure. The narrow $\pi$ band of the HOPG surface and the very localized F- $2 s$ band of $\mathrm{AlF}_{3}$ are clues for understanding the large energy electron emission in the collision of $\mathrm{He}^{+}$with both surfaces $[25,26]$. The strongly localized F- $2 s$ band allows for the neutralization of $\mathrm{He}^{+}$and therefore, the creation of long-lived holes in this band [26]. On the other hand, the emission of energetic electrons extending up to $30 \mathrm{eV}$ is explained by including the first excited state of helium in the collision of $\mathrm{He}^{+}$with a HOPG surface [25]. Both the charge fractions of the projectile and the energy distribution of electrons in the solid after the collision are calculated by using the appropriate Keldysh Green functions and the method of equations of motion (EOM). The electronic correlation in the localized projectile state is also taken into account for calculating the electron distribution in the solid, thus representing an improvement with respect to previous works $[18,25,26]$.

The paper is organized as follows: in Sec. II the theoretical framework is presented, while in Sec. III we introduce the several scattering situations related to the different scatter atoms considered: $\mathrm{K}$, a first neighbor $\mathrm{C}$ atom, a second neighbor $\mathrm{C}$ atom, and a carbon far enough as to resemble one of the pristine graphene surfaces. In this same section the projectile charge fractions, the energy distribution of the electrons in the surface after the collision, and the energy spectra of emitted electrons are presented and discussed in the range of incoming energies explored ( 0.5 to $2 \mathrm{keV})$. Finally, Sec. IV is devoted to the concluding remarks.

\section{THEORY}

In a previous work [18] we have presented in detail the theoretical treatment of the scattering of protons by a graphene surface with a low coverage of potassium atom impurities. Therefore, only a summary of the theoretical framework employed is presented below. The Anderson Hamiltonian [27] is the appropriate one for describing the three possible charge states of an $s$-valence hydrogen atom interacting with a solid surface. In the projection operators language, the Hamiltonian is written as [28]

$$
\begin{aligned}
\hat{H}= & \sum_{\vec{k} \sigma} \varepsilon_{\vec{k}} \hat{n}_{\vec{k} \sigma}+E_{0}|0\rangle\left\langle 0\left|+E_{1} \sum_{\sigma}\right| \sigma\right\rangle\left\langle\sigma\left|+E_{2}\right| \uparrow \downarrow\right\rangle\langle\uparrow \downarrow| \\
& +\sum_{\vec{k} \sigma}\left[V_{\vec{k} a}^{(1) \sigma} \hat{c}_{\vec{k} \sigma}^{\dagger}|0\rangle\left\langle\sigma\left|+V_{\vec{k} a}^{(1) \sigma *}\right| \sigma\right\rangle\langle 0| \hat{c}_{\vec{k} \sigma}\right] \\
& +\sum_{\vec{k} \sigma}(-1)^{P_{\sigma}}\left[V_{\vec{k} a}^{(2) \sigma} \hat{c}_{\vec{k} \sigma}^{\dagger}|\bar{\sigma}\rangle\left\langle\uparrow \downarrow\left|+V_{\vec{k} a}^{(2) \sigma *}\right| \uparrow \downarrow\right\rangle\langle\bar{\sigma}| \hat{c}_{\vec{k} \sigma}\right] .
\end{aligned}
$$

The first term of Eq. (1) refers to the energy eigenvalues of the system graphene plus an adsorbed potassium atom, which has been previously solved by considering the electronic correlation effects on the $\mathrm{K}$ site $[18,24]$. The three following terms represent the total energy of the possible charge configurations of the hydrogen atom: $|0\rangle,|\sigma\rangle$, and $|\uparrow \downarrow\rangle$, which correspond to zero, one with spin $\sigma$, and two electrons, respectively. The remaining terms describe the resonant charge exchange between the surface and the atomic states.

The hopping integral $V_{\vec{k} a}^{(1) \sigma}\left(V_{\vec{k} a}^{(2) \sigma}\right)$ is the coupling between the $\vec{k}$-surface state and the $a$ orbital of the projectile (1s for hydrogen atom), the supraindex 1 or 2 indicates coupling with the ionization and affinity energy levels, respectively. The total energies $E_{n}(n=0,1,2)$ for each electronic configuration are related to the ionization energy of the impurity atom $\varepsilon_{I}$, and with the intrasite electronic Coulomb repulsion $U$ in the $s$-valence orbital in the following way:

$$
\begin{gathered}
E_{1}-E_{0}=\varepsilon_{I}, \\
E_{2}-E_{0}=2 \varepsilon_{I}+U .
\end{gathered}
$$

In Eq. (1) the antisymmetry of the two electron wave function is taken into account through $P_{\sigma}=0$ if $\sigma=\uparrow$ and $P_{\sigma}=1$ if $\sigma=\downarrow$.

The correct normalization of the subspace including the selected atomic configurations is

$$
|0\rangle\left\langle 0\left|+\sum_{\sigma}\right| \sigma\right\rangle\langle\sigma|+| \uparrow \downarrow\rangle\langle\uparrow \downarrow|=\hat{1} .
$$

We are interested in calculating the ion neutralization probability

$$
P^{0}(t)=\sum_{\sigma}\left\langle\hat{n}_{1 \sigma}(t)\right\rangle=\sum_{\sigma}\langle\mid \sigma\rangle\langle\sigma \mid\rangle_{t}
$$

the negative ion formation probability

$$
P^{-}(t)=\left\langle\hat{n}_{2}(t)\right\rangle=\langle\mid \uparrow \downarrow\rangle\langle\uparrow \downarrow \mid\rangle_{t},
$$

and the energy distribution of the electrons in the solid

$$
N(\varepsilon, t)=\sum_{\vec{k} \sigma}\left\langle\hat{n}_{\vec{k} \sigma}\right\rangle_{t} \delta\left(\varepsilon-\varepsilon_{\vec{k}}\right)
$$

The time evolution of these all quantities, Eqs. (5), (6), and (7), are calculated by considering the equation of motions in the Heisenberg picture, $d \hat{A}(t) / d t=i[\hat{H}, \hat{A}(t)]$. The following 
equations result (atomic units are used):

$$
\begin{aligned}
& \frac{d\left\langle\hat{n}_{1 \sigma}(t)\right\rangle}{d t}=-\operatorname{Im} \sum_{\vec{k}}\left[V_{\vec{k} a}^{(1) *}(t) \int_{t_{0}}^{t} d \tau V_{\vec{k} a}^{(1) \sigma}(\tau)\left\{F_{\sigma}(\tau, t)-\left[2\left\langle\hat{n}_{\vec{k} \sigma}\left(t_{0}\right)\right\rangle-1\right] G_{\sigma}(\tau, t)\right\} e^{-i \varepsilon_{\vec{k}}(t-\tau)}\right. \\
&\left.-(-1)^{P_{\vec{\sigma}}} V_{\vec{k} a}^{(2) *}(t) \int_{t_{0}}^{t} d \tau V_{\vec{k} a}^{(2) \sigma}(\tau)\left\{F_{\uparrow \downarrow}(\tau, t)-\left[2\left\langle\hat{n}_{\vec{k} \sigma}\left(t_{0}\right)\right\rangle-1\right] G_{\uparrow \downarrow}(\tau, t)\right\} e^{-i \varepsilon_{\vec{k}}(t-\tau)}\right], \\
& \frac{d\left\langle\hat{n}_{2}(t)\right\rangle}{d t}=- \operatorname{Im} \sum_{\vec{k} \sigma}(-1)^{P_{\sigma}} V_{\vec{k} a}^{(2) *}(t) \int_{t_{0}}^{t} d \tau V_{\vec{k} a}^{(2) \sigma}(\tau)\left\{F_{\uparrow \downarrow}^{\bar{\sigma}}(\tau, t)-\left[2\left\langle\hat{n}_{\vec{k} \bar{\sigma}}\left(t_{0}\right)\right\rangle-1\right] G_{\uparrow \downarrow}^{\bar{\sigma}}(\tau, t)\right\} e^{-i \varepsilon_{\vec{k}}(t-\tau)}, \\
& \frac{d N(\varepsilon, t)}{d t}=\operatorname{Im} \sum_{\vec{k} \sigma}\left[V_{\vec{k} a}^{(1) *}(t) \int_{t_{0}}^{t} d \tau V_{\vec{k} a}^{(1) \sigma}(\tau)\left\{F_{\sigma}(\tau, t)-\left[2\left\langle\hat{n}_{\vec{k} \sigma}\left(t_{0}\right)\right\rangle-1\right] G_{\sigma}(\tau, t)\right\} e^{-i \varepsilon_{\vec{k}}(t-\tau)}\right. \\
&\left.+(-1)^{P_{\vec{\sigma}}} V_{\vec{k} a}^{(2) *}(t) \int_{t_{0}}^{t} d \tau V_{\vec{k} a}^{(2) \sigma}(\tau)\left\{F_{\uparrow \downarrow}(\tau, t)-\left[2\left\langle\hat{n}_{\vec{k} \sigma}\left(t_{0}\right)\right\rangle-1\right] G_{\uparrow \downarrow}(\tau, t)\right\} e^{-i \varepsilon_{\vec{k}}(t-\tau)}\right] \delta\left(\varepsilon-\varepsilon_{\vec{k}}\right) .
\end{aligned}
$$

The advanced Green functions $G_{\sigma}(\tau, t)$ and $G_{\uparrow \downarrow}(\tau, t)$, and $F_{\sigma}(\tau, t)$ and $F_{\uparrow \downarrow}(\tau, t)$, that appear in Eqs. (8), (9), and (10) are defined as

$$
\begin{aligned}
G_{\sigma}\left(t, t^{\prime}\right) & =i \Theta\left(t^{\prime}-t\right)\left\langle\left\{|\sigma\rangle\left\langle\left. 0\right|_{t^{\prime}} ; \mid 0\right\rangle\left\langle\left.\sigma\right|_{t}\right\}\right\rangle,\right. \\
G_{\uparrow \downarrow}^{\sigma}\left(t, t^{\prime}\right) & =i \Theta\left(t^{\prime}-t\right)\left\langle\left\{|\uparrow \downarrow\rangle\left\langle\left.\sigma\right|_{t^{\prime}} ; \mid \sigma\right\rangle\left\langle\left.\uparrow \downarrow\right|_{t}\right\}\right\rangle,\right. \\
F_{\sigma}\left(t, t^{\prime}\right) & =i\left\langle\left[|\sigma\rangle\left\langle\left. 0\right|_{t^{\prime}} ; \mid 0\right\rangle\left\langle\left.\sigma\right|_{t}\right]\right\rangle,\right. \\
F_{\uparrow \downarrow}^{\sigma}\left(t, t^{\prime}\right) & =i\left\langle\left[|\uparrow \downarrow\rangle\left\langle\left.\sigma\right|_{t^{\prime}} ; \mid \sigma\right\rangle\left\langle\left.\uparrow \downarrow\right|_{t}\right]\right\rangle,\right.
\end{aligned}
$$

where [;] and $\{;\}$ indicate commutator and anticommutator, respectively, and $\left\langle\hat{n}_{\vec{k} \sigma}\left(t_{0}\right)\right\rangle$ is the Fermi distribution. These Green functions are also calculated by the EOM method closed up to a second order in the atom-surface hopping term, as it is detailed in Ref. [18].

The energy distribution of electrons in the solid calculated by Eq. (10) contemplates the electronic repulsion in the projectile site, thus representing an improvement with respect to the calculation presented in Ref. [18].

\section{RESULTS AND DISCUSSION}

\section{A. Scattering process}

We study the scattering of protons by a graphene surface with a $\mathrm{K}$ atom adsorbed on a hollow site at a distance of 4.9 a.u. [18,24]. In the present work we analyze the scattering by carbon atoms which are neighbors of the $\mathrm{K}$ adsorbate and compare with the scattering by the $\mathrm{K}$ atom that was already thoroughly studied in a previous work [18]. In Fig. 1 we show the scatter sites of interest: a first $\mathrm{K}$ neighbor indicated by $\mathrm{C}_{1}$ and a second $\mathrm{K}$ neighbor by $\mathrm{C}_{2}$. The distances $\mathrm{K}-\mathrm{C}_{1}$ and $\mathrm{K}-\mathrm{C}_{2}$ are 5.6 and 7.3 a.u., respectively. A carbon atom far enough from the adsorbate site, as to be equivalent to a $\mathrm{C}$ atom of a clean graphene surface, is also considered as a reference (this is referred to as $\mathrm{C}$ and it is not shown in Fig. 1).

The ion trajectory is assumed perpendicular respect to the graphene surface (as shown in Fig. 1) with a constant velocity $z_{\text {in(out) }}=z_{\text {rtp }}+v_{\text {in(out) }} t$. The kinetic energy loss factor due to the elastic scattering of hydrogen by either a $\mathrm{K}$ atom or a $\mathrm{C}$ atom, and for a scattering angle equal to $180^{\circ}$, are 0.90 and 0.71 , respectively. These values are calculated consistently with the elastic binary collision model [29]. The analyzed incident ion energies are between 0.5 and $2 \mathrm{keV}$.
In the scattering by $\mathrm{K}$, the distances of closest approach are obtained from the interaction energy of the H-K dimer [18]. The calculated values vary from 0.18 a.u. for $2 \mathrm{keV}$ to 0.42 a.u. for $0.5 \mathrm{keV}$.

In Fig. 2(a) it is observed the large growth of the level width in the region of short distances to the surface $(<1$ a.u. $)$, in the scattering by a carbon atom close to the adsorbate. These features of the atom-surface interaction make very difficult and time consuming the numerical calculation of the distance evolution of the ion fractions in the region near to the surface, for turning points calculated from the projectile-scatter atom interaction energy. The region of distances effective for the charge exchange process can be determined from the characteristic times of the scattering process [30]: (i) the interaction time $\tau_{i}$ related to the inverse of the Anderson hybridization width $\Gamma_{a}, \tau_{i} \approx \frac{1}{2 \Gamma_{a}}$ and (ii) the collision time closely related to the time-energy uncertainty relation $\tau_{c} \approx \frac{1}{2 \Delta E_{v}}$, with $\Delta E_{v} \approx$ $\frac{v_{\perp}}{2}\left(v_{\perp}\right.$ is the ion velocity component perpendicular to the surface). For the charge exchange to effectively take place, both characteristic times should be comparable, $\tau_{c} \approx \tau_{i} \Rightarrow$ $\Gamma_{a} \approx \Delta E_{v}$. That is, the same order of magnitude for both times is required: $\frac{\Gamma_{a}}{\Delta E_{v}} \leqslant 10$ or $\frac{\Delta E_{v}}{\Gamma_{a}} \leqslant 10$.

Consequently, the defined spatial region where the charge exchange is efficient changes with the incoming projectile energy, suggesting in this way a consistent effective turning point. These spatial regions are shown in Fig. 2(a) for the maximum and minimum values of the ion velocity range analyzed in the present work. In this figure we have considered the hydrogen ionization level in the case of scattering by $\mathrm{C}_{1}$, whose calculation is discussed further in Sec. III B 1. From this figure we can infer effective distances of closest approach for hydrogen scattered by $\mathrm{C}_{1}$ that vary from 1.6 a.u. in the case of an incoming energy of $2 \mathrm{keV}$ to 2.0 a.u. for an incoming energy of $0.5 \mathrm{keV}$. For the scattering by either $\mathrm{C}_{2}$ or the carbon atom $\mathrm{C}$ far enough from $\mathrm{K}$, the same turning points are practically obtained.

The characteristic times of the collision allow for a determination of the effective region of distances where the charge transfer is well defined, providing in this form an alternative criterion for determining effective turning points that avoid the numerically conflictive region close to the surface. Of course the same final ion fractions must be obtained by using one 

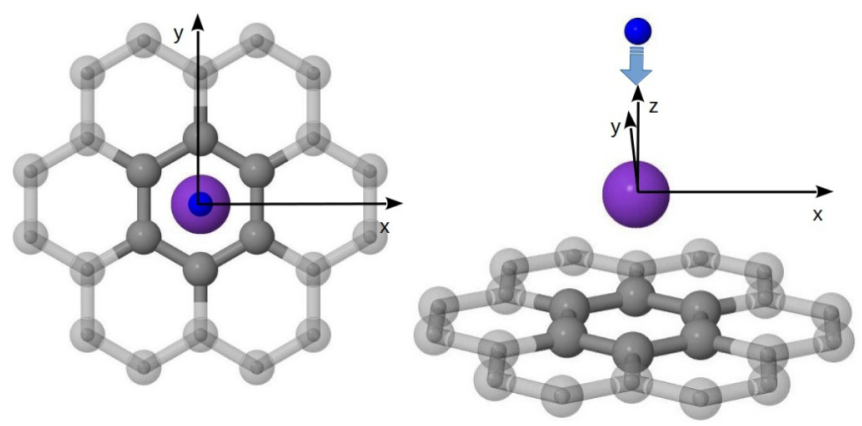

(a) $\mathrm{H}^{+}$scattered by $\mathrm{K}$.
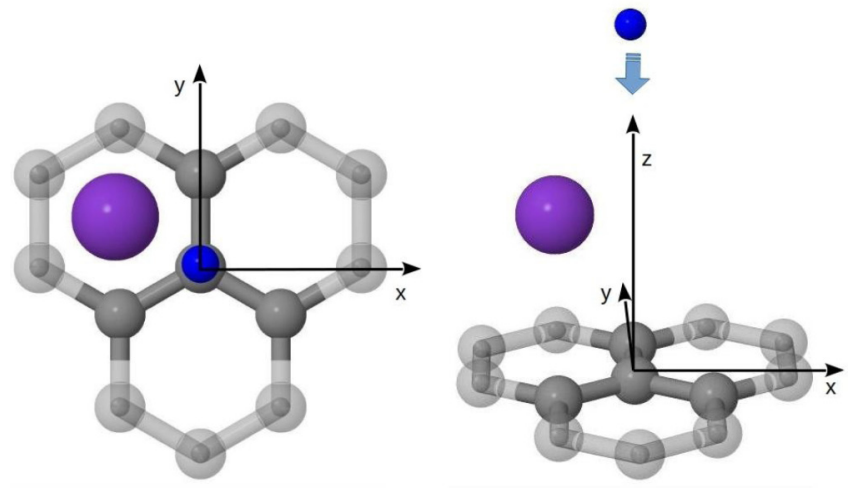

(b) $\mathrm{H}^{+}$scattered by $\mathrm{C}_{1}$.
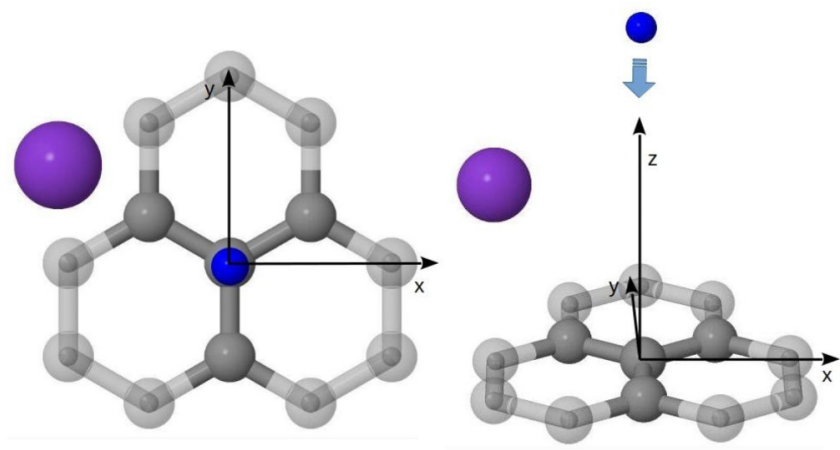

(c) $\mathrm{H}^{+}$scattered by $C_{2}$.

FIG. 1. Top and lateral view of $\mathrm{H}^{+}$in a frontal collision with different scatter sites. (a) $\mathrm{K}$-scatter site, (b) $\mathrm{C}_{1}$-scatter site, and (c) $\mathrm{C}_{2}$-scatter site. Big purple sphere corresponds to $\mathrm{K}$ atom; gray spheres correspond to $\mathrm{C}$ atoms; and small blue sphere corresponds to $\mathrm{H}$ projectile. The $(0,0,0)$ position is fixed at the scatter site in each case.

or another of the criteria. In Fig. 2(b) the charge fractions as a function of ion-surface distance are shown by using the turning point obtained either from the $\mathrm{H}-\mathrm{C}$ interaction energy or from the characteristic times $\left(\tau_{c} \approx \tau_{i}\right)$. It can be observed that the difference found between both calculations is less than 0.01 , verifying in this form that the characteristic times provide a good criterion for calculating effective turning points.

\section{B. Hamiltonian parameters}

The surface-atom coupling terms $V_{\vec{k} a}^{(j) \sigma}$, with $j=1,2$, and the total energy of the selected configurations $E_{n}$, involved in
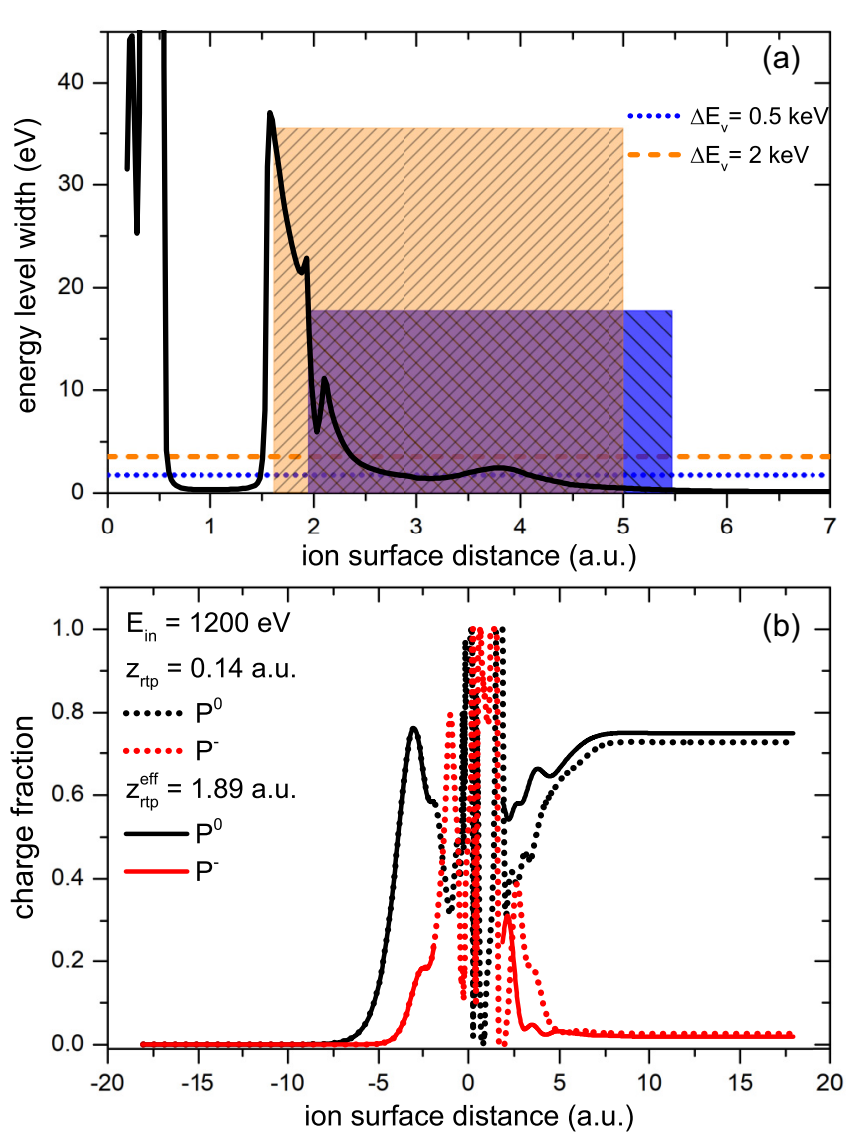

FIG. 2. (a) Comparison of the level widths due to the interaction $\Gamma_{a}$ and to the velocity $\Delta E_{v}$ for the ionization projectile energy level in the case of dispersion by $\mathrm{C}_{1} . \Delta E_{v}$ is plotted for the maximum ( $2 \mathrm{keV}$, orange) and minimum ( $0.5 \mathrm{keV}$, blue) incoming energies explored. The shadowed boxes for both limits of the explored energy range represent the spatial region where the charge transfer is effective and the criterion used to establish the box size is $\frac{\Delta E_{v}}{10} \leqslant$ $\Gamma_{a} \leqslant 10 \Delta E_{v}$. (b) Proton scattering by the $\mathrm{C}_{1}$ carbon atom. Negative (red lines) and neutral (black line) charge fractions as a function of the distance to the surface (negative distances for incoming trajectory, positive distances for outgoing trajectory), for an incoming energy $E_{\text {in }}=1200 \mathrm{eV}$. Dotted lines correspond to the turning point determined from the $\mathrm{H}-\mathrm{C}$ interaction energy and full lines to the one determined from the characteristic collision times.

Eq. (1), are calculated by using the bond pair model [31,32]. In this model a good calculation of the Hamiltonian parameters requires an atomic basis set that properly describes the properties of atoms, and an appropriate description of the surface electronic structure based on a LCAO expansion of the band states. The one electron hopping term $V_{\vec{k} a}^{(j) \sigma}$ is determined by performing a mean field approximation together with an overlap expansion of the many-body Hamiltonian. In this form, $V_{\vec{k} a}^{(j) \sigma}$ is finally recovered as a superposition of the atomic (dimeric) hopping integrals $V_{i \vec{R}_{s}, a}^{(j)}$ between the atomic states $\phi_{i \vec{R}_{s}}$ of an atom of the surface and the atomic state $\phi_{a}$ of the projectile. The final expression is

$$
V_{\vec{k} a}^{(j) \sigma}=\sum_{i \vec{R}_{s}} c_{i \vec{R}_{s}}^{\vec{k} \sigma} V_{i \vec{R}_{s}, a}^{(j)} .
$$


The coefficients $c_{i \vec{R}_{s}}^{\vec{k} \sigma}$ in Eq. (12) are related to the LCAO expansion of the $\vec{k}$-surface states, and they determine the elements of the density matrix of the solid given by

$$
\rho_{\sigma, i, j, \vec{R}_{s}, \vec{R}_{s}^{\prime}}^{(S)}(\varepsilon)=\sum_{\vec{k}} c_{i \vec{R}_{s} *}^{\vec{k} \sigma} c_{j \vec{R}_{s}^{\prime}}^{\vec{k}} \delta\left(\varepsilon-\varepsilon_{\vec{k} \sigma}\right)
$$

The complete density matrix of graphene plus potassium impurity was calculated and thoroughly discussed in Ref. [18]. The local density of states on $\mathrm{K}$ and on the $\mathrm{C}$ atoms involved in the scattering process, either as a scatter atom or as a near neighbor of it, is shown in Fig. 3.

The LDOS on the $\mathrm{K}$ site includes the $4 s$-valence orbital [24] and the $3 p$-core orbital $(-16 \mathrm{eV}$ referred to the Fermi energy [20,33]). The $3 p$ energy level falls within the valence band of graphene, and then it turns out to be widened by the interaction. The $3 s$-core state $(-32.4 \mathrm{eV}$ referred to the Fermi energy [33]) is also included as an inner band of zero width [not shown in Fig. 3(a)]. The K-4s LDOS, treated by considering the electronic correlation effects, has two very narrow peaks near the Fermi level and other two wider structures, one close to $-20 \mathrm{eV}$ and the other to $-7.5 \mathrm{eV}$ [see Fig. 3(a)] which reflect the mixing with graphene band states. In Fig. 3(b) the local densities of states on the $\mathrm{C}$ sites modified by the presence of K impurity are shown. We can see more clearly in Fig. 3(c) the perturbation produced by the presence of the impurity, which is notable at the energy positions of the main peaks found in the K LDOS and tends to disappear for $\mathrm{C}$ atoms farer than the second neighbors of $\mathrm{K}$ atom.

\section{Hydrogen energy levels}

Figure 4 shows the distance evolution of the ionization $\varepsilon_{I}=E_{1}-E_{0}$ and affinity $\varepsilon_{A}=\varepsilon_{I}+U$ hydrogen energies, referred to the Fermi level. These levels are obtained when hydrogen is scattered by $\mathrm{K}, \mathrm{C}_{1}, \mathrm{C}_{2}$, and $\mathrm{C}$ atoms. The pronounced downshift observed at short distances is mainly due to the attractive electron-nucleus interaction, while the large distance behavior is determined by the following potential:

$$
V_{e p}(z)=\frac{q}{r_{1}}-\frac{q}{r_{2}} \pm \frac{1}{4\left(z-z_{\mathrm{im}}\right)} .
$$

In Eq. (14) the first two terms account for the dipole potential formed by the adsorbate and its image, while the last term is originated in the image of the hydrogen ion projectile (the \pm signs corresponds to the positive/negative hydrogen ions). The charge of the alkaline atom is $q=0.1 e, r_{1}$ is the distance between the proton and the $\mathrm{K}$ adatom, and $r_{2}$ is the distance between the proton and the image charge of the positive charged $\mathrm{K}[18,24]$. The image plane position $z_{\mathrm{im}}$ for the graphene is 3.16 a.u. The potential given by Eq. (14) is introduced for distances larger than 8 a.u. with respect to the graphene surface, and for $z<8$ a.u. the energy levels are shifted in a constant value equal to $V_{e p}(z=8$ a.u.), in the same way that it was introduced in the image potential due to the ionic projectile [31,32].

As we can observe from Fig. 4, the effect of the adsorbate on the distance dependence of the projectile energy level is only visible when the dispersion is by the nearest $C$ atom $\left(C_{1}\right)$. In this case, for distances to the graphene surface between 2
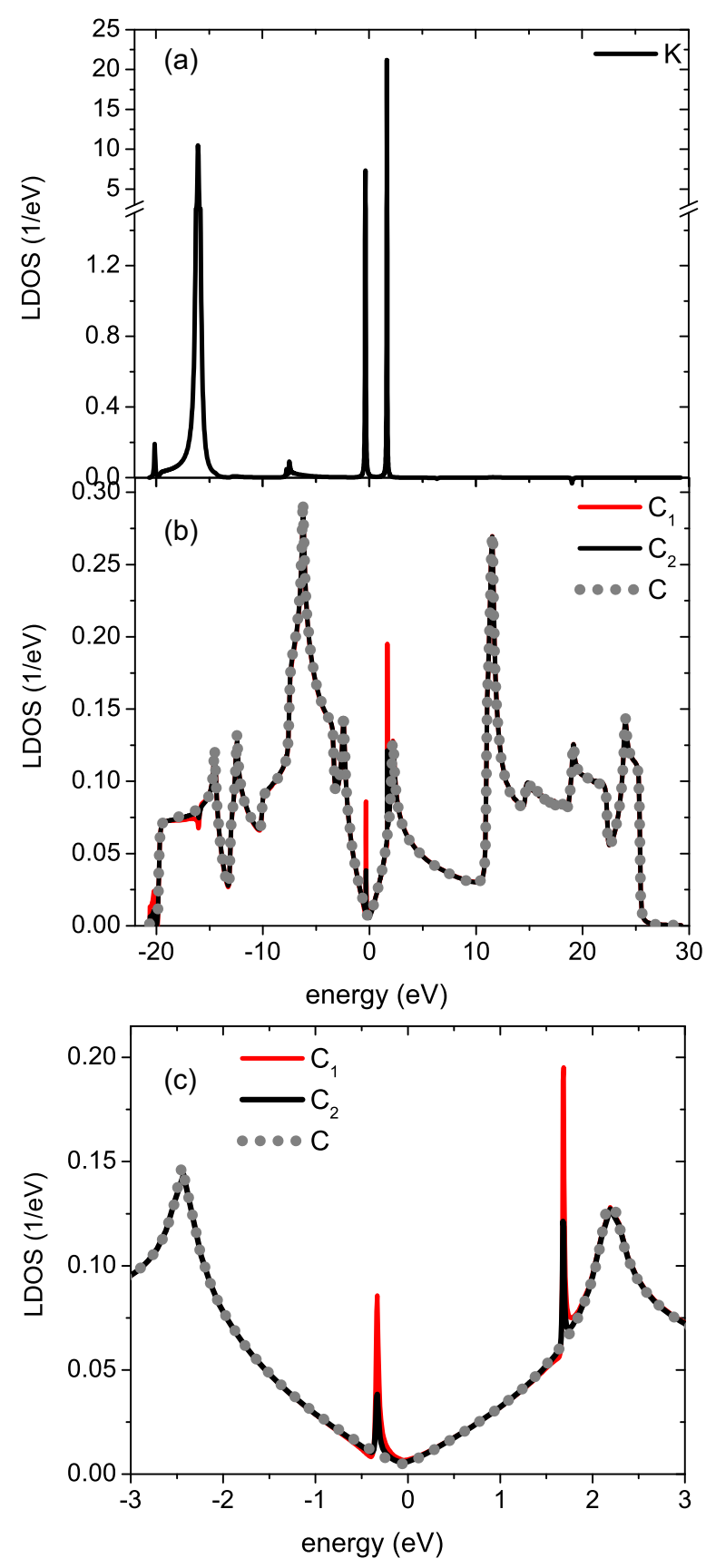

FIG. 3. Local density of states (LDOS) on the different atoms involved in the analyzed scattering processes shown in Fig. 1: (a) $\mathrm{K}$ atom (the break in the ordinate axis is to give visibility to the smaller LDOS structures), (b) $\mathrm{C}_{1}, \mathrm{C}_{2}$, and $\mathrm{C}$ atoms, and (c) a zoom in of (b) to view the details around the Fermi level.

and 9 a.u., the interaction with $\mathrm{K}$ introduces an oscillatory behavior in both ionization and affinity levels.

\section{Atom-atom couplings}

The atom-atom coupling $V_{i \vec{R}_{s}, a}^{(j)}$ between the hydrogen atom and the different scatter sites are shown in Fig. 5. It is also included in the coupling terms with the neighbors of the scatter site considered in each case. 


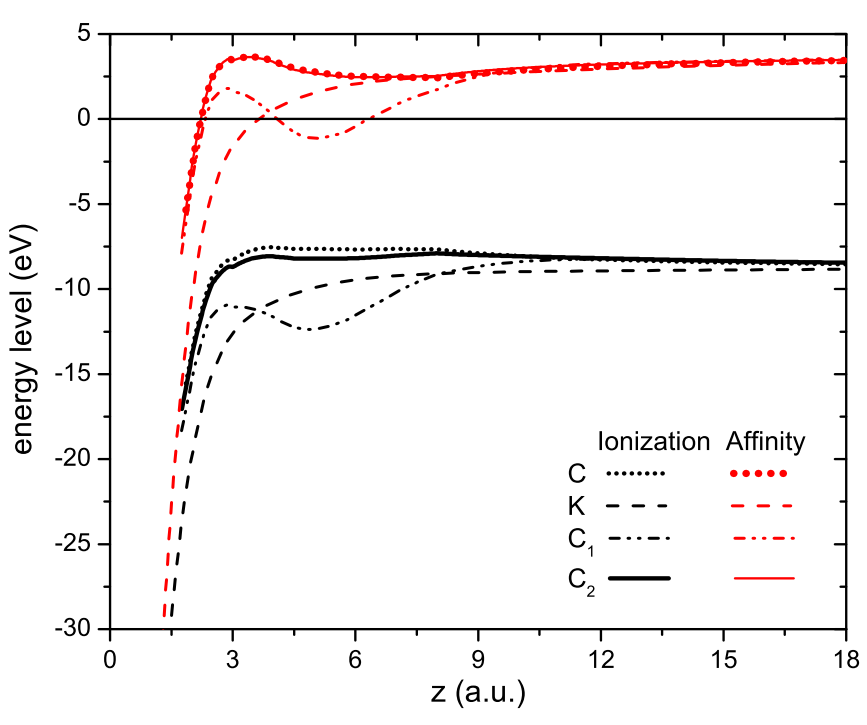

FIG. 4. Distance evolution of the ionization and affinity levels of hydrogen for different scatter sites. Dotted line corresponds to $\mathrm{C}$-scatter site, dashed line to $\mathrm{K}$-scatter site, dash-dotted-dotted line to $\mathrm{C}_{1}$, and solid line to $\mathrm{C}_{2}$.

In the frontal collision of hydrogen with potassium, the interaction between $\mathrm{H}$ and the $\mathrm{C}$ neighbors is negligible, as it is observed in Fig. 5(a). In the scattering by the carbon atom $\mathrm{C}_{1}$, the interaction with the valence $(4 s)$ and inner $(3 s$ and $3 p$ ) states of $\mathrm{K}$ is dominant for distances larger than 4 a.u. [Fig. 5(b)]. In the case of the scatter site $C_{2}$ [Fig. 5(c)], for distances lower than 5 a.u., the coupling with the $\mathrm{C}$ states is defining the $\mathrm{H}$-surface interaction, while for larger distances the extended coupling with the $4 s$-valence state of $\mathrm{K}$ is the dominant one.

From the hopping integrals behavior observed in Fig. 5, we can ensure that the chosen neighbors of the scatter site in each case are practically enough for calculating the expansion required in Eq. (12).

\section{Final charge states of the projectile}

The final neutral and negative charge fractions as a function of incoming ion energy are shown in Fig. 6 for the different scatter sites indicated in Fig. 1. The results presented in the case of hydrogen scattered by the $\mathrm{K}$ atom have been already published and discussed in Ref. [18]. The oscillatory behavior observed in this case is caused by the correlated states of hydrogen interacting with the localized band states of the potassium. When the scatter site is a carbon atom first neighbor of $\mathrm{K}\left(\mathrm{C}_{1}\right)$, the oscillations become attenuated due to the enabled interaction with the more extended band states of graphene (see Fig. 5). The marked diminution of the negative ion fraction in this last case is due to the empty band of graphene that enhances the possibility of electron loss. The energy dependence of the charge fractions in the scattering by a $C$ atom second neighbor of $K\left(C_{2}\right)$ is very similar to the smoother one obtained in the scattering by a $\mathrm{C}$ atom of a pristine graphene surface.

The neutral and negative ion fractions for the scattering sites $\mathrm{C}_{2}$ and $\mathrm{C}$ are very similar for energies below $1.5 \mathrm{keV}$,

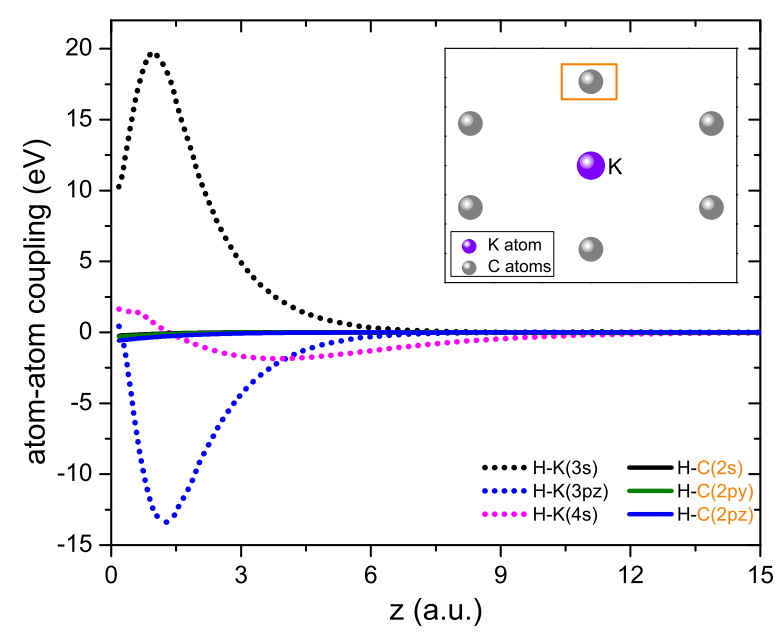

(a) $\mathrm{H}$ scattered by $\mathrm{K}$.

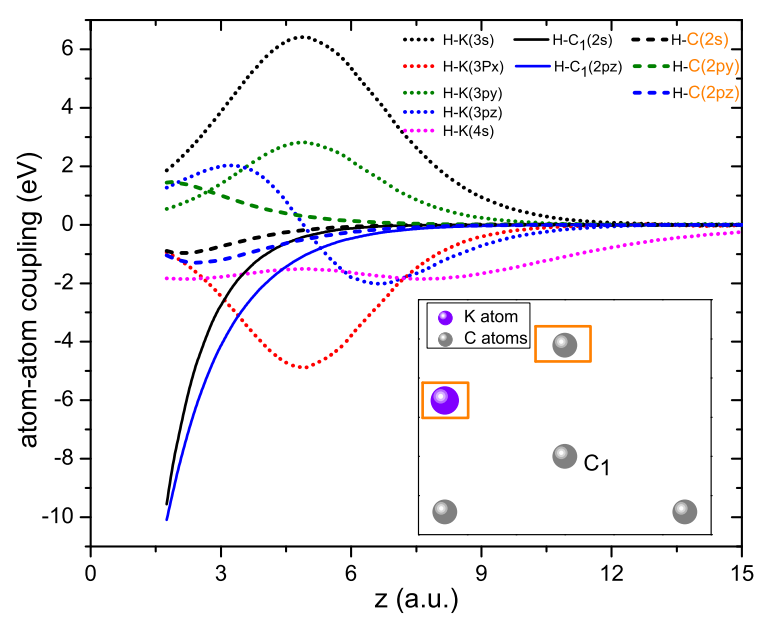

(b) $\mathrm{H}$ scattered by $\mathrm{C}_{1}$.

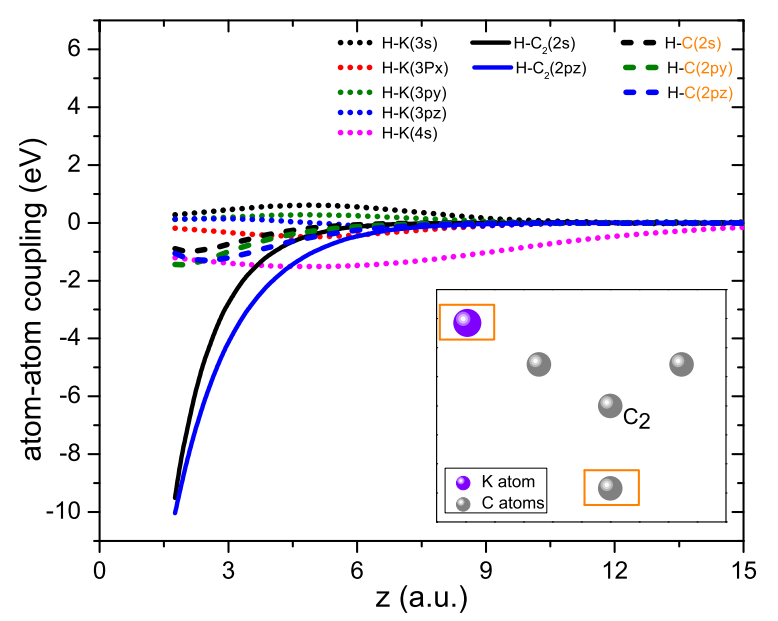

(c) $\mathrm{H}$ scattered by $C_{2}$.

FIG. 5. Atom-atom couplings $V_{i \vec{R}_{s}}^{(1)}$ as a function of normal distance $z$ to the surface, for the different scatter sites according to Fig. 1. (a) $K$, (b) $C_{1}$, and (c) $C_{2}$. In the respective insets, the scatter atom and the neighbors involved are shown (the nonequivalent are enclosed by orange squares).

while differences are appreciated for the largest energy values analyzed. As it is shown in Fig. 7, the effective region for the charge exchange is defined far from the surface at low ion 


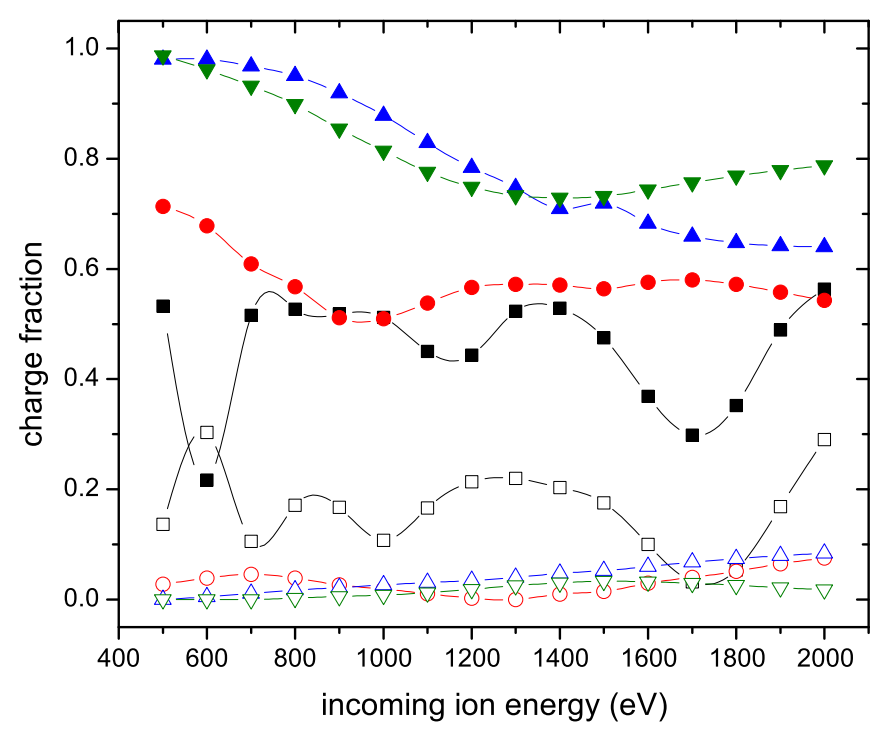

FIG. 6. Neutral (full symbol) and negative (open symbol) ion fractions as a function of the incoming ion energy when $\mathrm{H}^{+}$is scattered by $\mathrm{K}$ (black symbols), by $\mathrm{C}_{1}$ (red symbols), by $\mathrm{C}_{2}$ (blue symbols), and by $\mathrm{C}$ (green symbols).

velocities and it is closer as the incoming energy grows. Then, for low energies, the origin of the differences between the scattering by either $\mathrm{C}_{2}$ or $\mathrm{C}$ is mainly the interaction with the $4 s$ band states of $\mathrm{K}$, which becomes possible in the scattering by $\mathrm{C}_{2}$, as it is shown in Fig. 5(c). While for the largest values of incoming energy for which the effective charge exchange

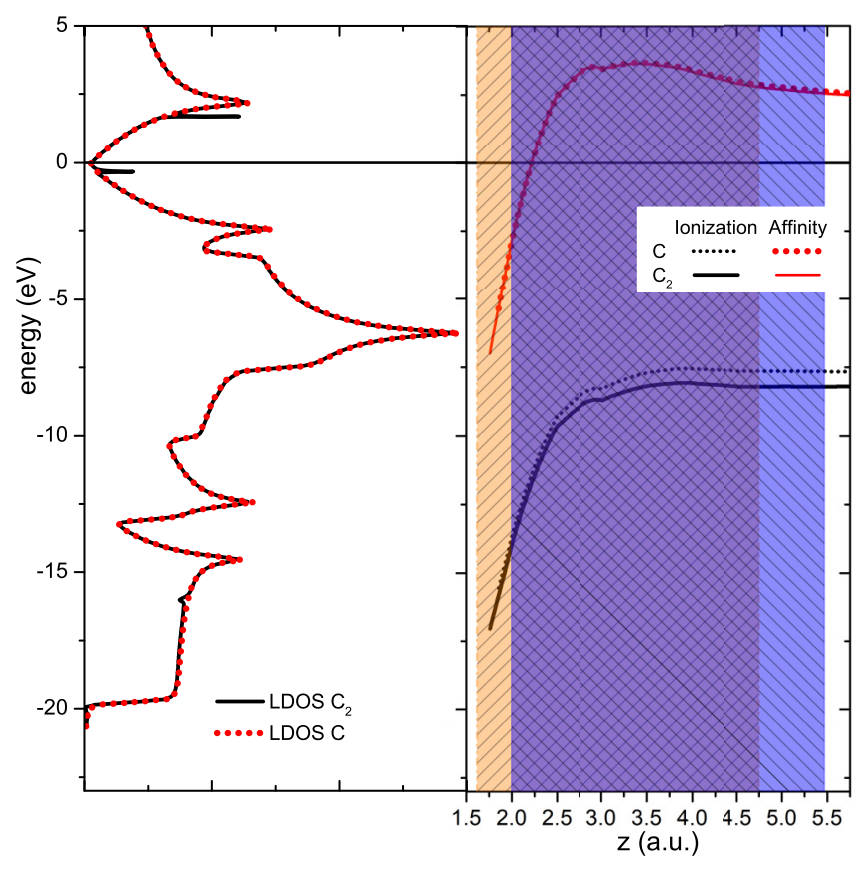

FIG. 7. Ion energy levels as a function of the distance to the graphene surface, confronted with the LDOS projected on the scatter site for both cases: proton scattered by either $\mathrm{C}_{2}$ or $\mathrm{C}$ atom (see Fig. 1). The effective charge exchange region of distances for incoming energies equal to 600 (blue box) and $1900 \mathrm{eV}$ (orange box) are shown in the same way as that in Fig. 2. region occurs close to the surface, the interaction with the carbon states becomes predominant, as it is also observed in Fig. 5(c). Then the peak under the Fermi level introduced by the presence of the adsorbate in the $\mathrm{C}_{2}$-LDOS (see Fig. 7) increases the possibility of negative ion formation for high velocities, compared with the case of proton scattering by $\mathrm{C}$. Thus, the changes in the density of states projected on $\mathrm{C}_{2}$ due to the interaction with the potassium adsorbate (also shown in Fig. 7) are, to a great extent, the responsible of the differences in the charge state fractions obtained for both scatter sites, $\mathrm{C}_{2}$ and $\mathrm{C}$.

The energy dependence of the charge fractions in the scattering by a $\mathrm{C}$ atom second neighbor of $\mathrm{K}\left(\mathrm{C}_{2}\right)$ is similar to the smoother one obtained in the scattering by a $\mathrm{C}$ atom of a pristine graphene surface, which indicates that for atoms farer than $C_{2}$ the presence of $K$ does not affect the charge transfer process, showing the localized effect of alkaline adsorbates also seen previously in other substrates $[15,16]$.

A nonmonotonous energy dependence of the charge transfer was found in the scattering from pristine graphene and from HOPG [23,34]. In these projectile/surface systems: $\mathrm{Li} /$ graphene and $\mathrm{H} / \mathrm{HOPG}$, the charge fractions dependence on the incoming energy are related to the suppression of the hybridization width. Also, a nonmonotonous dependence of charge transfer versus energy has been found in different projectile-target combinations, where the dependence is attributed to the presence of a projected band gap and surface states within it [35-41].

In the present work the more pronounced oscillatory energy behavior found in the case of the scattering by a carbon atom in the presence of a $\mathrm{K}$ adatom, compared with the case of a carbon atom in a pristine graphene, is directly related to the localized electronic states introduced by the weakly hybridized potassium in the density matrix of the combined surface K/graphene.

\section{Electron and hole distribution in the graphene surface with a low coverage of potassium}

We calculate the energy distribution of holes below the top of the valence band after the collision as the difference between the initial electron distribution at $t=-\infty$ and the final one at $t=\infty$ [Eq. (7)], $N_{h}(\varepsilon, \infty)=N(\varepsilon,-\infty)-N(\varepsilon, \infty)$ for $\varepsilon<\varepsilon_{F}$, and the energy distribution of excited electrons $N(\varepsilon, \infty)$, that is for energies above the Fermi level $\varepsilon>\varepsilon_{F}$. Both of them are shown in Fig. 8 for all the scatter sites considered according to Fig. 1: K, $\mathrm{C}_{1}, \mathrm{C}_{2}$, and C. For clarity, the hole distribution is shown in one panel and the distribution of excited electrons is shown in a second panel which corresponds to the conduction band region for energies above the Fermi level. The neutralization of the ion projectile and the excitation of the electrons in the solid are responsible for the holes that appear in the valence band.

The energy spectra of holes and electrons reflect fundamentally the characteristics of the local density of states of the potassium covered surface (see Fig. 3). Then, when the scattering is by the $\mathrm{K}$ atom, the hole and electron energy distribution observed in Fig. 8(a) show localized structures consistent with the $3 p$ and $4 s$ bands of the LDOS on potassium. The very narrow spectra of excited electrons follows the 

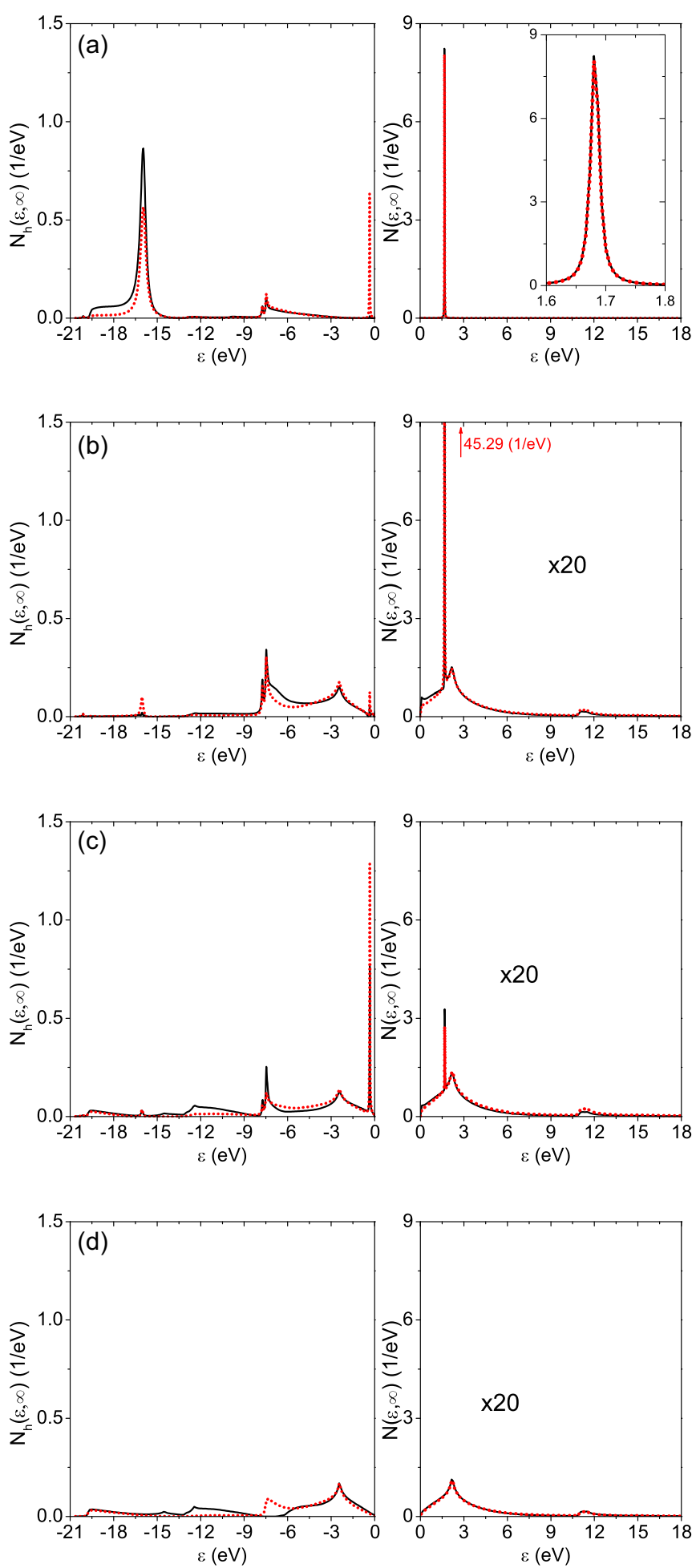

FIG. 8. Energy distribution of holes $N_{h}(\varepsilon, \infty)$ and the energy distribution of electrons excited to the conduction band $N(\varepsilon, \infty)$ for different incoming ion energy (black: $1 \mathrm{keV}$; red: $2 \mathrm{keV}$ ), for the four scatter sites considered: (a) $\mathrm{K}$, (b) $\mathrm{C}_{1}$, (c) $\mathrm{C}_{2}$, and (d) C. The excited electron spectra of (b), (c), and (d) are enhanced 20 times.

extremely localized nature of the empty $\mathrm{K}-4 s$ band. While in the scattering by a first neighbor $\mathrm{C}$ atom $\left(\mathrm{C}_{1}\right)$, Fig. 8(b), the $\sigma$ and $\pi$ bands of graphene begin to have an important presence on the hole energy distribution, and also in the broadened

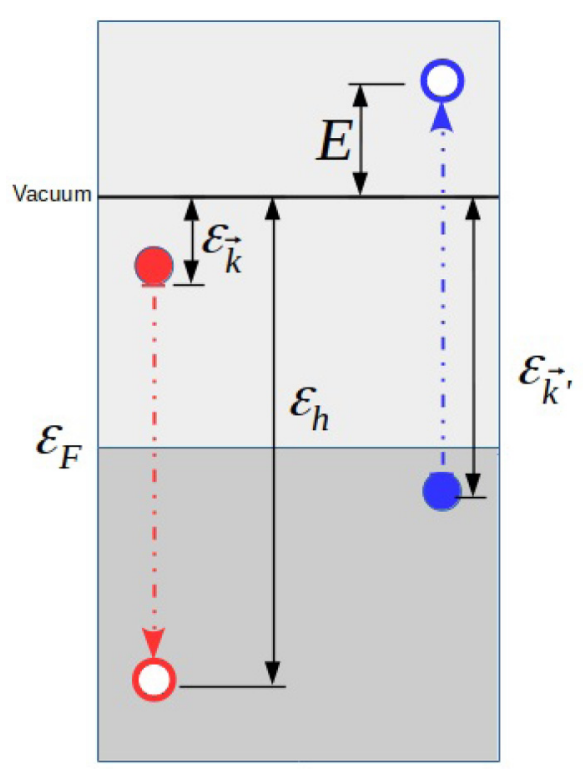

FIG. 9. The autoionization process is shown schematically. An initial excited electron with energy $\varepsilon_{\vec{k}}$ decays to the hole state with energy $\varepsilon_{h}$; then an electron with energy $\varepsilon_{\vec{k}^{\prime}}$ is emitted to a final state with energy $E=\varepsilon_{\vec{k}}+\varepsilon_{\vec{k}^{\prime}}-\varepsilon_{h}$.

spectra of excited electrons. In addition, the possibility of holes close to the bottom of the band diminishes due to the relative small contribution of the K-3p states. Narrow and prominent peaks appear due to the presence of the adsorbed $\mathrm{K}$ atom [see Fig. 3(b)], introducing in this form the signals of a nearby impurity in the energy distribution of electrons in the solid after an ion-surface collision. These signals are less remarkable as the scattering is from a $\mathrm{C}$ atom farther from the $\mathrm{K}$ atom [Fig. 8(c)], thus tending to the more extended energy spectra obtained in the case of the scattering by a $\mathrm{C}$ atom of a pristine graphene [Fig. 8(d)]. In the case of the scattering by $\mathrm{C}_{2}$, the charge exchange process is dominated by the interaction with the $\mathrm{C}$ atoms. The changes in the LDOS due to the presence of $\mathrm{K}$ (Fig. 3) lead to a larger neutralization than for the scattering by either $\mathrm{K}$ or $\mathrm{C}_{1}$ (see Fig. 6). Then the prominent peak that appears close to the Fermi level in the holes distribution can be related to the narrow localized structures in the LDOS of $\mathrm{C}_{2}$ and the surrounding carbon neighbors (see Figs. 1 and 3 ). While in the case of scattering by $\mathrm{C}_{1}$, the direct strong interaction with the $\mathrm{K}$ adatom along the ion trajectory dominates the charge exchange process. At the analyzed incoming energies, the neutralization is very similar to that in the case of scattering by $\mathrm{K}$ and smaller than in the case of scattering by $\mathrm{C}_{2}$ (see Fig. 6). Then the peak close to the Fermi level in the holes distribution [Fig. 8(c)] is mainly related to the contribution of the $\mathrm{K}-4 s$ band.

\section{E. Energy spectra of emitted electrons}

It is interesting, based on the energy distributions shown in Fig. 8, to analyze the autoionization decay spectra, where the energy of recombination of a hole and an electron is transferred to another electron in the valence band $[26,42]$. The basic idea behind the electron emission from the surface is schematically shown in Fig. 9. 
The energy distribution of emitted electrons as a function of the energy $N E(E)$ is given by

$$
\begin{aligned}
N E(E)= & \sum_{\vec{k}, \vec{k}^{\prime}, h} N\left(\varepsilon_{\vec{k}}, \infty\right) N\left(\varepsilon_{\vec{k}^{\prime}}, \infty\right) N\left(\varepsilon_{h}, \infty\right) \\
& \times T(E) \delta\left(E-\varepsilon_{\vec{k}}-\varepsilon_{\vec{k}^{\prime}}+\varepsilon_{h}\right),
\end{aligned}
$$

where all the energies are referred to the vacuum level $(E=0) ; \delta\left(E-\varepsilon_{\vec{k}}-\varepsilon_{\vec{k}^{\prime}}+\varepsilon_{h}\right)$ ensures the energy conservation in the emission process (see Fig. 9); and $T(E)$ corresponds to the transmission probability of the quantum step, given by

$$
T(E)=\frac{4 \sqrt{E} \sqrt{E+U_{0}}}{2 E+U_{0}+2 \sqrt{E} \sqrt{E+U_{0}}},
$$

$U_{0}=25.67 \mathrm{eV}$ is the potential barrier (width of the valence band plus work function). The emitted electron spectra $N E(E)$ given by Eq. (15) is shown in Fig. 10 for each scattering situation.

In the case of protons scattered by the $\mathrm{K}$ adsorbate, an intense emission of electrons with energies below $15 \mathrm{eV}$ is obtained for the whole range of incoming energies explored. The origin of these electron emission is the very narrow peak of excited electrons limited only to the K- $4 s$ empty band [see Fig. 8(a)]. When the scattering is by a $\mathrm{C}$ atom, the extended features of the $\sigma$ and $\pi$ bands of graphene lead to broader energy distributions of emitted electrons. The emission of electrons with larger energies (up to $20-25 \mathrm{eV}$ ) is made possible by the energy distribution of excited electrons broadened thanks to the extended empty band states of graphene. In the case of the scattering by $\mathrm{C}_{1}$, the intense emission of electrons of low energies (below $5 \mathrm{eV}$ ) is caused by both the $\mathrm{K}-4 s$ empty band and the perturbed empty local density of states on $\mathrm{C}_{1}$ by the potassium. While in the scattering by $\mathrm{C}_{2}$, the direct interaction with $\mathrm{K}$ is less possible, but the emission of low energy electrons is still more intense than in the case of a pristine graphene due to the signals of the adsorbed $\mathrm{K}$ atom on the $\mathrm{C}_{2}$ local density of states.

As it is observed in Fig. 10, the presence of the potassium atom is introducing a marked nonmonotonous dependence of the electron emission on the projectile incoming energy. This fact has the same origin as that of the oscillatory energy dependence of the charge fractions: the localized band structures introduced by the interaction of the adsorbate with the graphene surface.

As an overview of Sec. III, in LEIS experiments it is possible to determine if the proton was scattered by either $\mathrm{K}$ or $\mathrm{C}$ because of the mass difference that leads to elastic peaks well separated in energy [43]. For extremely low coverage of potassium, which is the case studied in the present work, the proton scattering by the adsorbate has really very little probability. But this is not the case of the scattering by carbon atoms, which is the object of study of this work. It is true that the charge fractions obtained from the analysis of the elastic peak, corresponding to the scattering by $\mathrm{C}$ atoms, correspond to the scattering by many $\mathrm{C}$ atoms, which differ in the distance to the adsorbate. Then the measured charge fractions will be a weighted average of the ones for different atoms such as $\mathrm{C}_{1}$, $\mathrm{C}_{2}$, and $\mathrm{C}$, discussed in the present work.

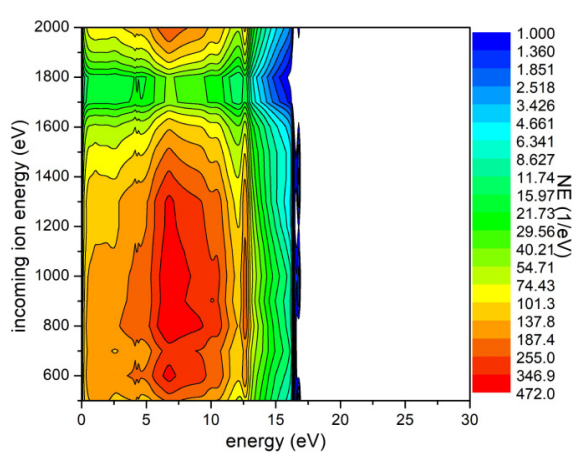

(a) K -site

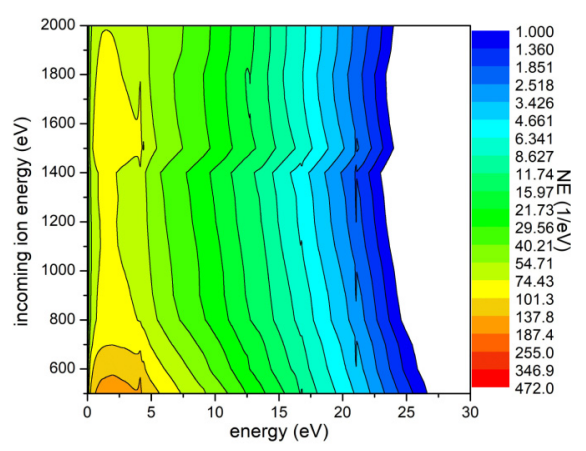

(b) $\mathrm{C}_{1}$-site

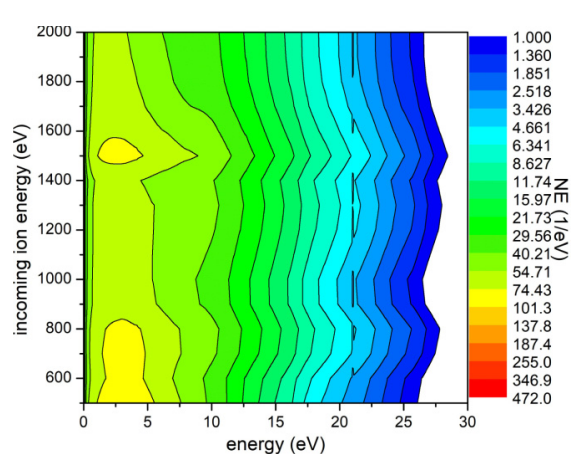

(c) $\mathrm{C}_{2}$-site

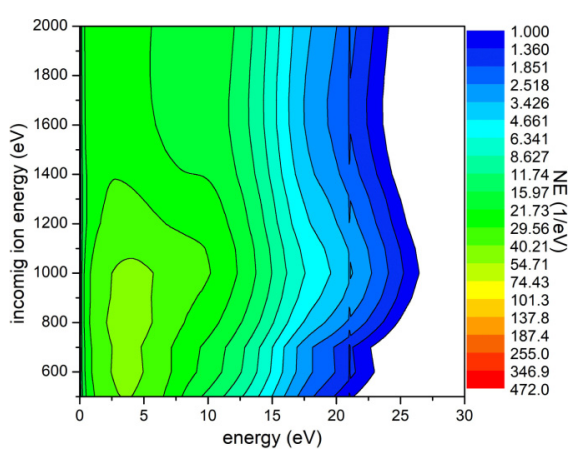

(d) C -site

FIG. 10. Surface contour plot: Energy distribution of emitted electrons for different incoming ion energies. The color scale is used to indicate $N E(E)$.

The usual measurements of emitted electron spectra are not performed in coincidence with the LEIS experiments, then it is not possible to relate them to the scattering by either $\mathrm{K}$ or $\mathrm{C}$ atoms. In our theoretical calculations, the ion-surface 
charge exchange and the electron emission occur in the same scattering process.

Nevertheless, our results surely allow for understanding the experimental results in the case of a very low coverage of potassium. As a first step in the study of different coverage, our work suggests the possible signals of the presence of adsorbates in the charge fractions and emitted electron spectra, when protons collide with a K/graphene surface. As a general conclusion, all these signals come from the localized features of the LDOS on the adsorbed alkali atom and on carbon atoms close to the adsorbate, introduced by the peculiarities of the electronic band structure of the graphene.

\section{CONCLUSIONS}

The charge fractions of protons, scattered by a graphene surface with a low coverage of potassium, have been theoretically analyzed when the binary collision occurs with different carbon atoms nearby the $\mathrm{K}$ adsorbate. The energy distribution of holes and excited electrons in the surface and the electron emission after the collision have also been calculated and discussed.

Electronic correlation effects were taken into account in the calculation of the density matrix of the graphene plus the adsorbate surface, and also in the charge exchange and electron emission that take place in the scattering of protons by this composed surface. The Anderson model Hamiltonian was used for describing the interacting system and the appropriate Keldysh Green functions for calculating the physical magnitudes of interest, such as the probability of the different possible charge states of the incoming projectile, and the energy distribution of the electrons that remain in the surface.

The oscillatory dependence of the hydrogen charge fractions with the incoming energy observed in the frontal collision with potassium [18], where the interaction with C atoms is negligible, becomes attenuated when the extended band states of graphene becomes more active in the charge exchange process. In the same way, the marked decrease of the negative ion fraction, compared to the scattering by $\mathrm{K}$, is caused by the increasing possibility of electron loss to the empty graphene bands. The extended band structure of graphene allows for the emission of electrons with larger energies (20-25 eV) while in the case of the collision with $\mathrm{K}$, the emitted electrons have energies below $15-17 \mathrm{eV}$, and that is because these electrons come from the recombination of holes with the excited electrons to the extremely localized $\mathrm{K}-4 s$ empty band states.

The signals of the localized structures introduced by the presence of potassium in the density matrix of the combined graphene plus adsorbate surface are evidenced in the intense emission of low energy electrons and also in the nonmonotonous dependence on the incoming projectile energy of both the charge exchange and the electron emission processes.

\section{ACKNOWLEDGMENTS}

The authors acknowledge financial support by Consejo Nacional de Investigaciones Científicas y Técnicas, Grant No. 0100546, Universidad Nacional del Litoral through the CAI+D Grant, and ANPCyT through Grant No. PICT-20164288.
[1] J. J. C. Geerlings, J. Los, J. P. Gauyacq, and N. M. Temme, Surf. Sci. 172, 257 (1986).

[2] H. Bonzel, A. Bradshaw, G. Ertl, D. W. H. H. und Else HeraeusStiftung, W.-H. Foundation, and G. Physikzentrum, Bad Honnef, Physics and Chemistry of Alkali Metal Adsorption, Materials science monographs (Elsevier, 1989).

[3] M. Ashwin and D. Woodruff, Surf. Sci. 244, 247 (1991).

[4] K. A. H. German, C. B. Weare, P. R. Varekamp, J. N. Andersen, and J. A. Yarmoff, Phys. Rev. Lett. 70, 3510 (1993).

[5] K. A. H. German, C. B. Weare, and J. A. Yarmoff, Phys. Rev. B 50, 14452 (1994).

[6] Q. B. Lu, R. Souda, D. J. O'connor, B. V. King, and R. J. MacDonald, Phys. Rev. B 54, R8389(R) (1996).

[7] Q. B. Lu, R. Souda, D. J. O'Connor, and B. V. King, Phys. Rev. Lett. 77, 3236 (1996).

[8] C. Weare and J. Yarmoff, Surf. Sci. 348, 359 (1996).

[9] A. Borisov, G. Makhmetov, D. Teillet-Billy, and J. Gauyacq, Surf. Sci. 375, L367 (1997).

[10] J. P. Gauyacq and A. G. Borisov, J. Phys.: Condens. Matter 10, 6585 (1998).

[11] M. Gleeson and A. Kleyn, Nucl. Instrum. Methods Phys. Res. B 157, 48 (1999).

[12] Y. Yang and J. A. Yarmoff, Phys. Rev. Lett. 89, 196102 (2002).

[13] Y. Yang, Z. Sroubek, and J. A. Yarmoff, Phys. Rev. B 69, 045420 (2004).
[14] X. Chen, Z. Sroubek, and J. Yarmoff, Surf. Sci. 602, 620 (2008).

[15] B. Bahrim, S. Yu, B. Makarenko, and J. Rabalais, Surf. Sci. 603, 703 (2009).

[16] S. Yu, B. Bahrim, B. Makarenko, and J. Rabalais, Surf. Sci. 606 1700 (2012).

[17] M. Rutigliano, A. Palma, and N. Sanna, Surf. Sci. 664, 194 (2017).

[18] M. A. Romero, A. Iglesias-García, E. A. García, and E. C. Goldberg, Phys. Rev. B 100, 085432 (2019).

[19] K. S. Novoselov, V. Fal, L. Colombo, P. Gellert, M. Schwab, K. Kim et al., Nature (London) 490, 192 (2012).

[20] K. T. Chan, J. B. Neaton, and M. L. Cohen, Phys. Rev. B 77, 235430 (2008).

[21] N. Dimakis, D. Valdez, F. A. Flor, A. Salgado, K. Adjibi, S. Vargas, and J. Saenz, Appl. Surf. Sci. 413, 197 (2017).

[22] W. Liu, C. Zhang, M. Deng, and S. Cai, Physica E 93, 265 (2017).

[23] M. A. Romero, A. Iglesias-García, and E. C. Goldberg, Phys. Rev. B 83, 125411 (2011).

[24] E. A. García, A. Iglesias-García, S. G. Carrillo, and M. A Romero, Appl. Surf. Sci. 452, 507 (2018).

[25] A. Iglesias-García, F. Bonetto, R. Vidal, J. Ferron, and E. C. Goldberg, Phys. Rev. A 89, 042702 (2014).

[26] A. Iglesias-García, C. González, and E. C. Goldberg, Phys. Rev. B 96, 075428 (2017). 
[27] P. W. Anderson, Phys. Rev. 124, 41 (1961).

[28] A. C. Hewson, The Kondo Problem to Heavy Fermions (Cambridge University Press, Cambridge, 1993).

[29] K. Oura, V. Lifshits, A. Saranin, A. Zotov, and M. Katayama, Surface Science: An Introduction (Springer Science \& Business Media, New York, 2013).

[30] M. Tacca, V. Quintero Riascos, R. A. Vidal, C. Gonzalez, F. Bonetto, and E. C. Goldberg, J. Phys. Chem. C 124, 3632 (2020).

[31] P. G. Bolcatto, E. C. Goldberg, and M. C. G. Passeggi, Phys. Rev. A 50, 4643 (1994).

[32] P. G. Bolcatto, E. C. Goldberg, and M. C. G. Passeggi, Phys. Rev. B 58, 5007 (1998).

[33] M. Cardona and L. Ley, Topics in Applied Physics, edited by M. Cardona and L. Ley (Springer, Berlin, 1978).

[34] F. J. Bonetto, M. A. Romero, A. Iglesias-García, R. A. Vidal, and E. C. Goldberg, J. Phys. Chem. C 119, 3124 (2015).

[35] A. R. Canario, T. Kravchuk, and V. Esaulov, New. J. Phys. 8, 227 (2006).
[36] A. Iglesias_García, E. A. Garcia, and E. C. Goldberg, J. Phys.: Condens. Matter 23, 045003 (2011).

[37] L. Gao, Y. Zhu, Y. Shi, P. Liu, Y. Xiao, G. Li, Y. Liu, V. A. Esaulov, X. Chen, L. Chen et al., Phys. Rev. A 96, 052705 (2017).

[38] V. Q. Riascos, M. Tacca, R. Vidal, C. Gonzalez, E. C. Goldberg, and F. Bonetto, J. Phys. Chem. C 122, 28192 (2018).

[39] Y. Shi, X. Zhang, Y. Li, P. Liu, B. Ding, L. Yin, Z. Zhang, Y. Zhu, X. Chen, Y. Guo et al., Appl. Surf. Sci. 487, 116 (2019).

[40] Y. Xiao, Y. Shi, P. Liu, Y. Zhu, L. Gao, Y. Guo, L. Chen, X. Chen, and V. A. Esaulov, Nucl. Instrum. Methods Phys. Res. Sect. B 450, 73 (2019).

[41] P. Liu, L. Yin, Z. Zhang, B. Ding, Y. Shi, Y. Li, X. Zhang, X. Song, Y. Guo, L. Chen et al., Phys. Rev. A 101, 032706 (2020).

[42] N. Bajales, L. Cristina, S. Mendoza, R. A. Baragiola, E. C. Goldberg, and J. Ferrón, Phys. Rev. Lett. 100, 227604 (2008).

[43] H. H. Brongersma, M. Draxler, M. de Ridder, and P. Bauer, Surf. Sci. Rep. 62, 63 (2007). 